\title{
Role of cyclooxygenase-2 in intestinal injury in neonatal rats
}

\author{
HUI $\mathrm{LU}^{1}$ and BING ZHU ${ }^{2}$ \\ Departments of ${ }^{1}$ Neonatology and ${ }^{2}$ Cardiac Surgery, Hangzhou Hospital, \\ Nanjing Medical University, Hangzhou, Zhejiang 310006, P.R. China
}

Received July 31, 2014; Accepted September 3, 2014

DOI: 10.3892/br.2014.362

\begin{abstract}
Necrotizing enterocolitis (NEC) is the most common gastrointestinal emergency in premature neonates. The pathogenesis of NEC remains poorly understood. The present study aimed to investigate the dynamic change and role of cyclooxygenase-2 (COX-2) in neonatal rats with intestinal injury. Wistar rats, $<24 \mathrm{~h}$ in age, received an intraperitoneal injection with $5 \mathrm{mg} / \mathrm{kg}$ lipopolysaccharide (LPS). Ileal tissues were collected at 1, 3, 6, 12 and $24 \mathrm{~h}$ following the LPS challenge for histological evaluation of NEC and for measurements of COX-2 mRNA. The correlation between the degree of intestinal injury and expression of $C O X-2$ mRNA was determined. The LPS-injected pups showed a significant increase in injury scores compared to the control, and the most deteriorating change was at $12 \mathrm{~h}$. $C O X-2 \mathrm{mRNA}$ expression was upregulated following LPS injection. There was a significantly positive correlation between $C O X-2 \mathrm{mRNA}$ and the grade of intestinal injury within $12 \mathrm{~h}$, whereas $C O X-2$ mRNA expression had a significantly negative correlation with the severity of intestinal injury at $24 \mathrm{~h}$. COX-2 plays an important role in LPS-induced intestinal injury and the repair processes. Caution should be exerted concerning the potential therapeutic uses of COX-2 inhibitors or promoters in NEC.
\end{abstract}

\section{Introduction}

Necrotizing enterocolitis (NEC) is one of the most damaging intra-abdominal emergencies in premature infants and is the cause of significant mortality and morbidity, including neurodevelopmental impairment, particularly in extreme preterm neonates requiring surgery for the illness (1-3). Although the pathogenesis of NEC remains elusive, the principal initiating events are believed to involve gut ischemia, formula feeding and intestinal colonization with opportunistic pathogens. These perinatal insults weaken the integrity of the immature

Correspondence to: Dr Hui Lu, Department of Neonatology, Hangzhou Hospital, Nanjing Medical University, 261 Huansha Road, Shangcheng, Hangzhou, Zhejiang 310006, P.R. China

E-mail: luhui6699@sina.com

Key words: cyclooxygenase-2, necrotizing enterocolitis, rat, newborn gut barrier, resulting in bacterial translocation and activation of innate immune responses (4).

Cyclooxygenase-2 (COX-2) is a rate-limiting enzyme in the synthesis of prostanoids from their precursor, arachidonic acid. Inhibitors of COX, such as glucocorticoids and nonsteroidal anti-inflammatory drugs, have been implicated as an NEC risk factor $(5,6)$. By contrast, COX-2 is proinflammatory and therefore, irregularly high COX-2 levels may be pathogenic during intestinal inflammation (7). These data indicate that COX-2 may play protective and deleterious roles in NEC. However, the exact role of COX-2 in the pathogenesis of NEC has not been fully elucidated. In a previous study of COX-2 in NEC, no correlation was found between COX-2 expression and intestinal injury severity (8).

Therefore, the aim of the present study was to investigate the dynamic change and the potential role of $C O X-2$ mRNA in neonatal rat with lipopolysaccharide (LPS)-induced intestinal injury, and to define whether NEC is associated with the expression of $C O X-2 \mathrm{mRNA}$ in the mucosa of the affected intestine tissue.

\section{Materials and methods}

Animal model. Wistar rats, $<24 \mathrm{~h}$ in age (mean weight, $6.24 \pm 0.81 \mathrm{~g}$ ), were administered an intraperitoneal (IP) injection of $5 \mathrm{mg} / \mathrm{kg}$ Escherichia coli $\mathrm{O}_{55}: \mathrm{B}_{5}$ endotoxin (LPS; Sigma-Aldrich, St. Louis, MO, USA) or a similar volume of saline (9-11). All the pups were sacrificed at 1, 3, 6, 12 or $24 \mathrm{~h}$ after receiving LPS IP $(n=8)$. The control pups $(n=8)$ were sacrificed at $1 \mathrm{~h}$ after saline IP. The pups that succumbed prior to the collection of the specimens were excluded from the study.

Specimens collection. All the surviving animals were sacrificed via decapitation. The gastrointestinal (GI) tract was carefully removed. The small intestine was subsequently divided into two halves: jejunum and ileum. A 3-cm segment of distal ileum, which was $4 \mathrm{~cm}$ proximal to the ileocecal valve, from each animal was cut and fixed for histological evaluation of NEC. The remainder of the ileum was snap-frozen at $-80^{\circ} \mathrm{C}$ for mRNA measurement.

\section{Experimental methods and analysis marker}

NEC evaluation. The segment of distal ileum was harvested, fixed in $4 \%$ paraformadehyde, embedded in paraffin, microtome-sectioned at $5 \mu \mathrm{m}$ and counterstained with hematoxylin 
and eosin for histological evaluation of intestinal injury. Histological changes in the ileum were scored by a blinded investigator and were assigned a NEC score on a scale $0-4$ as follows: 0 , normal, intact villous epithelium with normal histology; 1 , mild villous edema with epithelial sloughing confined to the tips of the villi; 2, mild midvillous necrosis; 3 , moderate midvillous necrosis with crypts still readily detectable; and 4 , severe necrosis of entire villi with complete absence of epithelial structures $(11,12)$.

Reverse transcription-polymerase chain reaction (RT-PCR) for COX-2 and $\beta$-actin. Total RNA was extracted using the Biotragents ${ }^{\mathrm{TM}}$ reagent (Sino-American Biotechnology, Co., Luoyang, China) and $2 \mu \mathrm{l}$ RNA was used to synthesize cDNA in the presence of an oligo dT 15-primer, RNase inhibitor and the avian myeloblastosis virus reverse transcriptase in a final volume of $20 \mu \mathrm{l}$. Sequence-specific oligonucleotide primers (Bioasia Biotechnology, Co., Ltd., Shanghai, China) were designed according to rat podocin as follows: $C O X-2$ sense, 5'-TTC AAA TGA GAT TGT GGG AAA ATT GCT-3'; and antisense, 5'-AGA TCA TCT CTG CCT GAG TAT CTT T-3' and $\beta$-actin sense, 5'-CAC CCT GTG CTG CTC ACC GAG GCC-3'; and antisense, 5'-CCA CAC AGA TGA CTT GCG CTC AGG-3'. The expected size of amplification was 305 base pairs (bp) for $C O X-2$ and $314 \mathrm{bp}$ for $\beta$-actin. PCR was performed in a $25-\mu \mathrm{l}$ reaction system, which contained $3 \mu \mathrm{l}$

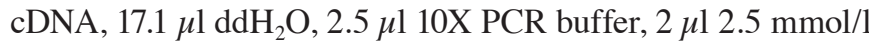
dNTPs, $0.2 \mu \mathrm{l}$ Taq DNA polymerase [Takara Biotechnology (Dalian), Co., Ltd., Dalian, China] and $0.1 \mu \mathrm{l}$ of each primer. Amplification cycles of $C O X-2$ were $95^{\circ} \mathrm{C}$ for $1.5 \mathrm{~min}$, followed by 45 cycles at $94^{\circ} \mathrm{C}$ for $45 \mathrm{sec}, 55^{\circ} \mathrm{C}$ for $45 \mathrm{sec}, 72^{\circ} \mathrm{C}$ for $1.5 \mathrm{~min}$ and terminated by a final extension of $72^{\circ} \mathrm{C}$ for $10 \mathrm{~min}$.

The PCR products were subjected to electrophoresis with $2 \%$ agarose gel and stained with ethidium bromide. The band intensity was determined by gel image analysis system (Kodak 1D; Eastman Kodak, Rochester, NY, USA). The relative mRNA concentrations were normalized for $\beta$-actin. The expression levels of $C O X-2$ mRNA were calculated by dividing the intensity of the internal control, $\beta$-actin.

Statistical analysis. Software SPSS 19.0 for Windows (SPSS, Inc., Chicago, IL, USA) was used in all the statistical tests. Comparisons between the groups were calculated using one-way analysis of variance and all the data are expressed as mean \pm standard deviation. $\mathrm{P}<0.05$, was considered to indicate a statistically significant difference. The degree of correlation was described using the Spearman's rank-correlation test.

\section{Results}

Incidence and severity of NEC. Using the histological scoring system, tissues with histological scores $\geq 2+$ were designated positive for NEC. In the LPS-injected group, 52.5\% (21/40) showed significant $(\mathrm{P}<0.01)$ pathological changes in ileal structure characterized as moderate (2+), severe (3+) or full necrosis (4+) compared to only a $0 \%(0 / 8)$ incidence of NEC in the control group. The most deteriorating change was at $12 \mathrm{~h}$ and the incidence of NEC was $87.5 \%$ (7/8). There was severe necrosis of the entire villi with complete absence of epithelial structures. The degree of ileal damage was also significantly
Table I. Scores of the lesion on distal ileum morphology and COX-2 mRNA of neonatal rats.

\begin{tabular}{lcc}
\hline Groups & Scores & COX-2 mRNA \\
\hline Control $(\mathrm{n}=8)$ & $0.12 \pm 0.17$ & $0.14 \pm 0.03$ \\
LPS $(\mathrm{n}=8)$ & & \\
$1 \mathrm{~h}$ & $1.28 \pm 0.62^{\mathrm{a}}$ & $0.25 \pm 0.04^{\mathrm{a}}$ \\
$3 \mathrm{~h}$ & $1.75 \pm 0.74^{\mathrm{a}}$ & $0.93 \pm 0.01^{\mathrm{a}}$ \\
$6 \mathrm{~h}$ & $1.98 \pm 0.75^{\mathrm{a}}$ & $1.01 \pm 0.07^{\mathrm{a}}$ \\
$12 \mathrm{~h}$ & $2.85 \pm 0.41^{\mathrm{a}}$ & $1.24 \pm 0.01^{\mathrm{a}}$ \\
$24 \mathrm{~h}$ & $2.35 \pm 0.63^{\mathrm{a}}$ & $1.35 \pm 0.08^{\mathrm{a}}$ \\
\hline
\end{tabular}

${ }^{\mathrm{a}} \mathrm{P}<0.05$ vs. control group. Data are mean \pm standard deviation. COX-2, cyclooxygenase-2; LPS, lipopolysaccharide.

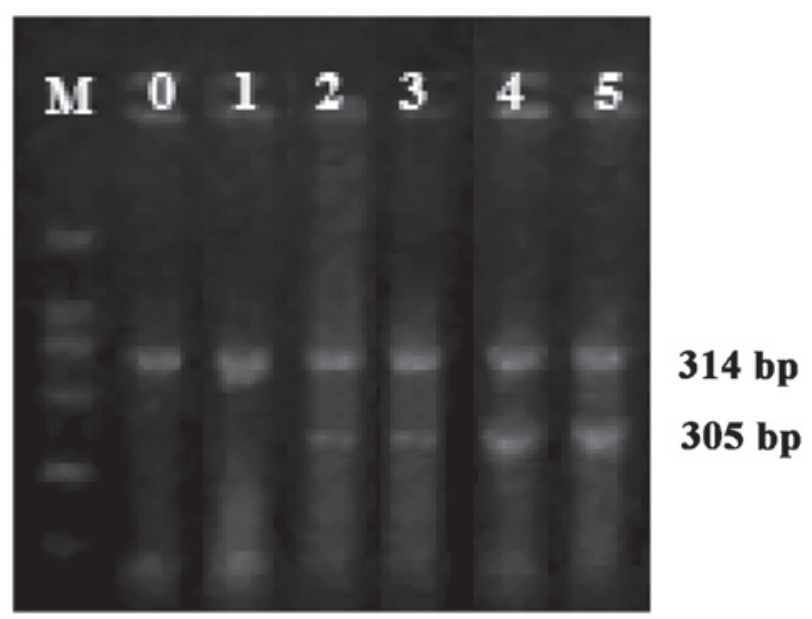

Figure 1. Gene expression of cyclooxygenase-2 in the ileum of neonatal rats. A total of $2 \%$ ethidium bromide stained agarose gel molecular marker (Kodak 1D) was used as an indicator. M, DNA marker DL2000; lane 0, control group; lane 1, lipopolysaccharide (LPS) $1 \mathrm{~h}$; lane 2, LPS 3 h; lane 3, LPS $6 \mathrm{~h}$; lane 4, LPS 12 h; lane 5, LPS 24 h; bp, basepair.

$(\mathrm{P}<0.05)$ increased in the LPS-injected compared to the control group (Table I).

Expression of COX-2 mRNA. The expression of COX-2 mRNA was significantly upregulated following LPS treatment $(\mathrm{P}<0.05)$ (Table I and Fig. 1). There was a significantly positive correlation between $C O X-2$ mRNA expression and the grade of intestinal injury at $1,3,6$ and $12 \mathrm{~h}(\gamma=0.892,0.855,0.770$ and $0.877 ; \mathrm{P}<0.05$ ). There was a significantly negative correlation between COX-2 mRNA and severity of NEC only at $24 \mathrm{~h}$ $(\gamma=-0.769, P<0.05)$.

\section{Discussion}

In the present study, significantly lower levels of $C O X-2$ mRNA were detected in the ileal tissue in a cohort of control rats. The COX-2 mRNA expression was significantly increased following LPS injection. There was a significantly positive correlation between the expression of $C O X-2 \mathrm{mRNA}$ and the degree of intestinal injury within $12 \mathrm{~h}$ after LPS 
injection. These results showed that an increased expression of ileal $C O X-2$ mRNA results in a significant increase of the incidence and severity of NEC. The $C O X-2$ mRNA expression was not significantly decreased at $24 \mathrm{~h}$, and there was a significantly negative correlation with the severity of intestinal injury.

NEC is predominately a disease of premature infants. In recent years, its incidence has become more prevalent with the increasing survival of low-birth weight premature infants (13). The pathogenesis of NEC continues to be investigated, however, the unifying hypothesis includes mucosal injury of the small intestine, followed by bacterial translocation and an amplified inflammatory response to endotoxin (14).

The COX enzymes are critical in the biosynthesis of prostanoids, play significant roles in the gut and are key for intestinal epithelium maintenance (15). COX, the enzyme that catalyzes the first two steps in the biosynthesis of the prostaglandins from arachidonic acid, exists in two isoforms. COX-1 is constitutively expressed throughout the GI tract and, at least in the absence of damage or inflammation, is the major source of prostaglandin synthesis in these tissues (16). The inducible form, COX-2, is either undetectable or expressed at extremely low levels in the healthy GI tract of humans and various animals (15). However, in response to various proinflammatory stimuli, COX-2 is rapidly induced. An increase in COX-2 protein expression was noted in the perforated intestinal sections of all 36 neonates examined in the study by Chung et al (13). High intestinal COX levels have been identified in an animal model of $\operatorname{NEC}(7,13)$.

COX-2 was initially regarded as a target for anti-inflammatory drugs. Suppression of the activity of this enzyme reduces edema formation and hyperalgesia. Therefore, COX-2 is also a major contributor to the processes that result in resolution of inflammation. A study of paw edema in COX-2-deficient and wild-type mice identified the significance of COX-2 in the resolution of inflammation (17). $C O X$-knockout mice are susceptible to intestinal disorders (18). The deficiency is correlated with enhanced intestinal epithelial permeability, which results in exaggerated bacterial translocation and increased mortality during peritonitis-induced sepsis (19).

Grosfeld et al (6) reported a cytoprotective role for prostaglandin E1, showing an increased NEC risk and bowel perforation in premature infants with patent ductus arteriosus (PDA) receiving indomethacin (INDO). Mortality was higher in the PDA/INDO group with NEC compared to the PDA/INDO infants without NEC. There was a significant association between toll-like receptor-4 (TLR4) signaling and $C O X-2$ expression in the gut. TLR4 and MyD88 signaling are required for optimal proliferation and protection against apoptosis in the injured intestine (17). MyD88 deficiency has been shown to aggravate intestinal ischemia/reperfusion injury and inhibit increases in $C O X-2$ expression and prostaglandin E2 synthesis during the development of injury (20). LPS-induced $C O X-2$ expression stimulates the proliferation of colonocytes and repair of colonic epithelium, therefore LPS stimulation of COX-2 was protective in experimental NEC (7). The expression of COX isoforms in the duodenum is upregulated by feeding and inhibition of COX-1 or COX-2 induces ulcers in the duodenum, indicating that the two isoforms play a critical role in the protection of the intestinal mucosa (21).
The outcome following the development of selective COX-2 inhibitors, with a purpose to reduce inflammation whilst sparing the GI tract from injury, was a series of discoveries that indicated a crucial role of COX-2 in GI mucosal defense and repair. There are low levels of $C O X-2$ expression in the healthy GI tract, however, it also significantly contributes to mucosal immunity and to the ability of the mucosa to resist injury induced by luminal irritants. The $C O X-2$ gene quickly responds to stress and the downstream products of this enzyme are potent lipid mediators that increase the resistance to injury and regulate the dynamics of inflammation and resolution (22).

Combined, these data indicate that a quick induction of $C O X-2$ is a general response to luminal irritation that is aimed at increasing mucosal resistance to injury and at priming for the preparation of mucosal repair in the event that injury does occur (22). Therefore, resolution of inflammation is a critical process in restoring homeostasis and COX-2 plays a crucial role in this process.

The present data showed that the expression of $C O X-2$ mRNA was significantly upregulated following LPS injection. The aforementioned studies indicate that COX-2 plays key roles in the ability of the GI mucosa to respond to injury. $C O X-2$ mRNA expression was significantly upregulated with the repair of intestinal injury at $24 \mathrm{~h}$, suggesting the induction of $C O X-2$ activity participates in the exacerbation of the injury and resolution of inflammation (23).

In conclusion, $C O X-2$ plays a significant role in neonatal rats with LPS-induced intestinal injury and repair processes. Caution should be exerted concerning the potential therapeutic uses of specific inhibitors or promoters of COX-2 at the optimal phase of inflammation and further information is required to define the role of the $\mathrm{COX} /$ prostaglandin pathway in the pathogenesis of NEC.

\section{References}

1. Stoll BJ, Hansen NI, Bell EF, et al; Eunice Kennedy Shriver National Institute of Child Health and Human Development Neonatal Research Network: Neonatal outcomes of extremely preterm infants from the NICHD Neonatal Research Network. Pediatrics 126: 443-456, 2010.

2. Lin PW and Stoll BJ: Necrotising enterocolitis. Lancet 368: 1271-1283, 2006.

3. Fanaroff AA, Stoll BJ, Wright LL, et al; NICHD Neonatal Research Network: Trends in neonatal morbidity and mortality for very low birthweight infants. Am J Obstet Gynecol 196: 147. e1-e8, 2007.

4. Emami CN, Chokshi N, Wang J, et al: Role of interleukin-10 in the pathogenesis of necrotizing enterocolitis. Am J Surg 203: 428-435, 2012.

5. Guthrie SO, Gordon PV, Thomas V, et al: Necrotizing enterocolitis among neonates in the United States. J Perinatol 23: 278-285, 2003.

6. Grosfeld JL, Chaet M, Molinari F, et al: Increased risk of necrotizing enterocolitis in premature infants with patent ductus arteriosus treated with indomethacin. Ann Surg 224: 350-357, 1996.

7. Bergholz R, Zschiegner M, Eschenburg G, et al: Mucosal loss with increased expression of IL-6, IL-8, and COX-2 in a formula-feeding only neonatal rat model of necrotizing enterocolitis. J Pediart Surg 48: 2301-2307, 2013.

8. Grishin AV, Wang J, Potoka DA, et al: Lipopolysaccharide induces cyclooxygenase-2 in intestinal epithelium via a noncanonical p38 MAPK pathway. J Immunol 176: 580-588, 2006.

9. Premer DM, Goertz R, Georgieff MK, et al: Muscle proteolysis and weight loss in a neonatal rat model of sepsis syndrome. Inflammation 26: 97-101, 2002. 
10. Qureshi FG, Leaphart C, Cetin S, et al: Increased expression and function of integrins in enterocytes by endotoxin impairs epithelial restitution. Gastroenterology 128: 1012-1022, 2005.

11. Lu H, Zhu B and Xue XD: Role of neuronal nitric oxide synthase and inducible nitric oxide synthase in intestinal injury in neonatal rats. World J Gastroenterol 12: 4364-4368, 2006.

12. Hammerman C, Goldschmidt D, Caplan MS, et al: Protective effect of bilirubin in ischemia-reperfusion injury in the rat intestine. J Pediatr Gastroenterol Nutr 35: 344-349, 2002.

13. Chung DH, Ethridge RT, Kim S, et al: Molecular mechanisms contributing to necrotizing enterocolitis. Ann Surg 233: 835-842, 2001.

14. Petrosyan M, Guner YS, Williams M, et al: Current concepts regarding the pathogenesis of necrotizing enterocolitis. Pediatr Surg Int 25: 309-318, 2009.

15. Kargman S, Charleson S, Cartwright M, et al: Characterization of prostaglandin $\mathrm{G} / \mathrm{H}$ synthase 1 and 2 in rat, dog, monkey, and human gastrointestinal tracts. Gastroenterology 111: 445-454, 1996.

16. Wallace JL, Bak A, McKnight W, et al: Cyclooxygenase 1 contributes to inflammatory responses in rats and mice: implications for gastrointestinal toxicity. Gastroenterology 115: 101-109, 1998

17. Fukata M, Chen A, Klepper A, et al: Cox-2 is regulated by Toll-like receptor-4 (TLR4) signaling: role in proliferation and apoptosis in the intestine. Gastroenterology 131: 862-877, 2006.
18. Ishikawa TO, Oshima M and Herschman HR: Cox-2 deletion in myeloid and endothelial cells, but not in epithelial cells, exacerbates murine colitis. Carcinogenesis 32: 417-426, 2011.

19. Fredenburgh LE, Velandia MM, Ma J, et al: Cyclooxygenase-2 deficiency leads to intestinal barrier dysfunction and increased mortality during polymicrobial sepsis. J Immunol 187: 5255-5267, 2011.

20. Watanabe T, Kobata A, Tanigawa T, et al: Activation of the MyD88 signaling pathway inhibits ischemia-reperfusion injury in the small intestine. Am J Physiol Gastrointest Liver Physiol 303: G324-G334, 2012.

21. Satoh H, Amagase K, Ebara S, et al: Cyclooxygenase (COX)-1 and COX-2 both play an important role in the protection of the duodenal mucosa in cats. J Pharmacol Exp Ther 344: 189-195, 2013.

22. Wallace JL and Devchand PR: Emerging roles for cyclooxygenase-2 in gastrointestinal mucosal defense. Br J Pharmacol 145: 275-282, 2005

23. Wallace JL: COX-2: a pivotal enzyme in mucosal protection and resolution of inflammation. Scientific World Journal 6: 577-588, 2006. 